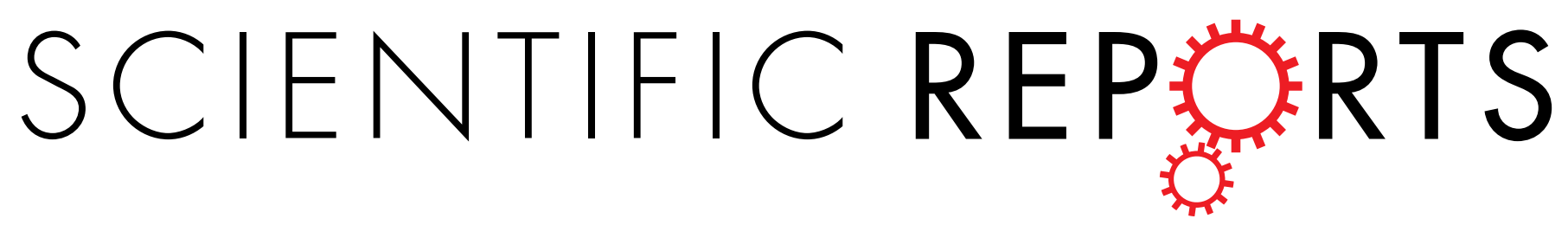

\title{
Corrigendum: Interfacial dominated ferromagnetism in nanograined ZnO: a $\mu$ SR and DFT study
}

Thomas Tietze, Patrick Audehm, Yu-Chun Chen, Gisela Schütz, Boris B. Straumal, Svetlana G. Protasova, Andrey A. Mazilkin, Petr B. Straumal, Thomas Prokscha, Hubertus Luetkens, Zaher Salman, Andreas Suter, Brigitte Baretzky, Karin Fink, Wolfgang Wenzel, Denis Danilov \& Eberhard Goering

Scientific Reports 5:8871; doi:10.1038/srep08871; published online 09 March 2015; updated 07 July 2015 This Article contains typographical errors in Equation 3:

$$
\mathrm{A}_{0} \cdot p_{Z F}(t)=A_{\text {Non }}+\underbrace{A_{\text {Fast }} \cdot \exp \left(-\lambda_{F} t\right)}_{\text {high field }}+\underbrace{A_{\text {Slow }} \cdot\left[\frac{1}{3}+\frac{2}{3}\left(\lambda_{S} t\right)^{-\alpha} \cdot \exp \left(-\frac{\left(\lambda_{S} t\right)^{-\alpha}}{\alpha}\right)\right]}_{\text {VKT, low field }}
$$

should read:

$$
A_{0} \cdot P_{Z F}(t)=A_{\text {Non }}+\underbrace{A_{\text {Fast }} \cdot \exp \left(-\lambda_{F} t\right)}_{\text {high field }}+\underbrace{A_{\text {Slow }} \cdot\left[\frac{1}{3}+\frac{2}{3}\left[1-\left(\lambda_{S} t\right)^{\alpha}\right] \cdot \exp \left(-\frac{\left(\lambda_{S} t\right)^{\alpha}}{\alpha}\right)\right]}_{\text {VKT, low field }}
$$

